\title{
亚纯函数结合于导数的总亏量 ${ }^{*}$ \\ 杨乐 \\ (中国科学院数学研究所, 北京 100080)
}

关镜调亚纯函数、亏量、分支指标

\section{一、Drasin 的几个问题}

设 $f(z)$ 是开平面上的超越亚纯函数, $k$ 为一正整数. $f(z)$ 的亏值与亏是是函数值分布 论中十分重要与基本的概念 ${ }^{(1,2)}$. 由于这时 $f^{(k)}(x)$ 也是超越亚纯函数, 于是它也可以有亏值 与亏量. 那么 $f(z)$ 的亏值与亏量和 $f^{(k)}(z)$ 的亏值与亏量间是否存在什么联系呢?

若 $f(x)$ 是有穷级整函数,则从 Nevanlinna 第二基本定理 ${ }^{[1,2}$ 易于得到

$$
\sum_{a \in c} \delta(a, f) \leqslant \delta\left(0, f^{(k)}\right),
$$

其中 $\sum_{a \in c} \delta(a, f)$ 表示 $f(\boldsymbol{z})$ 的全体有穷亏值的总亏量. 若 $f(\boldsymbol{x})$ 是有穷级亚纯函数, 则不难 证明

$$
\begin{aligned}
\sum_{a \in c} \delta(a, f) & \leqslant(k+1-\Theta(\infty, f)) \delta\left(0, f^{(k)}\right) \\
& \leqslant(k+1-\delta(\infty, f)) \delta\left(0, f^{(k)}\right),
\end{aligned}
$$

这里 $\Theta(\infty, f)$ 是值 $\infty$ 关于 $f(\boldsymbol{z})$ 的分支指标, 由

$$
\Theta(\infty, f)-1-\lim \sup _{r \rightarrow \infty} \frac{\bar{N}(r, f)}{T(r, f)}
$$

定义. 于是当有穷级亚纯函数 $f(z)$ 适合条件 $\sum_{a \in c} \delta(a, f)=2$ 时便有 $\delta\left(0, f^{\prime}\right)-1$. 一个 自然的问题是 $f^{\prime}(z)$ 还有其他亏值吗?

Drasin ${ }^{[3]}$ 研究了一些具体例子后, 提出了以下的猜测与问题.

(A) 若 $f(\boldsymbol{s})$ 是开平面上的有穷级亚纯函数, 且适合

$$
\sum_{a \in \mathrm{C}} \delta(a, f)=2 \text { 与 } \delta(\infty, f)-0,
$$

Drasin 猜测

$$
\sum_{b \in \mathrm{C}} \delta\left(b, f^{\prime}\right)=\delta\left(0, f^{\prime}\right)-1
$$

*国家自然科学基金资助项目. 
这里 $\sum_{a \in \mathrm{C}} \delta(a, f)$ 表示 $f(s)$ 的总亏量, $\sum_{b \in \mathrm{C}} \delta\left(b, f^{\prime}\right)$ 表示 $f^{\prime}(\boldsymbol{s})$ 的总亏量, 均包含值 $\infty$ 在内.

(B) 若 $f(x)$ 于开平面亚纯, 且 $8(\infty, f)-0$. 是否可能有

$$
\sum_{a \in C} \delta(a, f)+\sum_{b \in C} \delta\left(b, f^{\prime}\right)-4 ?
$$

（C）如果等式(2)不可能成立, 那么(2)式左端的最佳上界是什么?

\section{二、Drasin 问居的解答与有关结果}

它理 1 设 $f(x)$ 为开平面上的有穷级亚纯函数, $k$ 为一正整数, 则

$$
\sum_{a \in \mathrm{C}} \delta(a, f)-\sum_{b \in \mathrm{C}} \delta\left(b, f^{(k)}\right) \leqslant 3 \text {. }
$$

式中的等号当且仅当下述两种情况时成立:

(i) $\Theta(\infty, f)-1, \sum_{i \in \mathrm{C}} \delta(a, f)-1$ 与 $\sum_{b \in \mathrm{C}} \delta\left(b, f^{(k)}\right)-2$;

(ii) $k-1, \Theta(\infty, f)-0, \sum_{a \in \mathrm{C}} \delta(a, f)-2$ 与 $\sum_{b \in \mathrm{C}} \delta\left(b, f^{\prime}\right)-1$.

定理 1 中使等号成立的两种情况都是可以发生的.情况（i）的例子是函数 $e^{x}$; 情况（ii） 的例子有函数 $\frac{e^{x}}{e^{x}+1}$.

定理 1 结合熟知的不等式(1)便给予了 Drasin 的猜测 (A) 肯定的回答. 当 $f(x)$ 是有 穷级时, 它也给予问题 (B) 以否定的回答, 同时回答了问题 (C), 最佳上界等于 3.

如果在问题 (B) 中不假定 $\delta(\infty, f)-0$, 则等式 (2) 是可能成立的. 事实上, 我们有

定理 2 设 $f(x)$ 为开平面上的有穷级亚纯函数, $k_{0}$ 为一正整数. 为了使

$$
\sum_{a \in \mathrm{C}} \delta(a, f)+\sum_{b \in \mathrm{C}} \delta\left(b, f^{\left(k_{0}\right)}\right)-4,
$$

必要且充分的条件是

$$
\delta(\infty, f)-1, \quad \sum_{a \in \subset} \delta(a, f)-1
$$

与

$$
\delta\left(0, f^{(k)}\right)-\delta\left(\infty, f^{(k)}\right)-1(k-1,2, \cdots) .
$$

我们还可以将 Drasin 的猜测 (A) 提得更普遍些,并且给予肯定的回答.

定理 3 设 $f(z)$ 为开平面上的有穷级亚纯函数. 若 $\sum_{a \in c} \delta(a, f)-2$, 则

$$
\sum_{b \in \mathrm{C}} \delta\left(b, f^{(k)}\right)=\delta\left(0, f^{(k)}\right)=\frac{2}{k+1}(k-1,2, \cdots) .
$$

类似于定理 1 , 我们可以考虑亚纯函数的总亏量与其导数总亏量的其他类型的估计. 例 - 如

定理 4 设 $f(x)$ 为开平面上的有穷级亚纯函数, $k$ 为一正整数, 则

$$
\left(\sum_{a \in \mathbf{C}} \delta(a, f)\right)\left(\sum_{b \in \mathbf{C}} \delta\left(b, f^{(k)}\right)\right) \leqslant 2 .
$$




\section{三、Mues 符 测}

1971 年, Mues ${ }^{[4]}$ 提出了以下猜测:

设 $f(\boldsymbol{x})$ 于开平面超越亚纯, $k$ 为一正整数,则

$$
\sum_{a \in \mathrm{C}} \delta\left(a, f^{(k)}\right) \leqslant 1 \text {. }
$$

如果 $f(x)$ 适合条件 $N(r, f)=o\{T(r, f)\}$, 易知 Mues 猜测成立. 较一般地, 若 $j(x)$ 适合

$$
N(r, f)-k \bar{N}(r, f)-o\{T(r, f)\},
$$

则应用 Frank 与 Weissenborn ${ }^{[9]}$ 的一个引理, 也不难证明 Mues 猜测成立. 我们还可以考 虑其他很不相同的特殊情况,

定理 5 设 $f(x)$ 为开平面上的有穷级亚纯函数, $k$ 为一正整数. 若

$$
\theta(\infty, f) \leqslant \frac{\left(1-\frac{1}{k}\right)+\left(1+\frac{1}{k}\right) \delta\left(\infty, f^{(k)}\right)}{1+\delta\left(\infty, f^{(k)}\right)},
$$

则 Mues 猜测(即不等式(3))成立. 特别地, 若 $\Theta(\infty, f) \leqslant 1-\frac{1}{k}$, 则 Mues 猜测成立.

定理 6 设 $f(\boldsymbol{x})$ 为开平面上的有穷级亚纯函数, $k$ 为一正整数. 若

$$
\left(1+\delta\left(\infty, f^{(k)}\right)\right)\left(\sum_{a \in \mathrm{C}} \delta(a, f)\right) \geqslant 2,
$$

则 Mues 猜测成立. 特别地, 若

$$
\sum_{a \in \mathrm{C}} \delta(a, f)+\delta\left(\infty, f^{(k)}\right) \geqslant 2,
$$

则 Mues 猜测成立.

对于一般情况，Mues 猜测还未获得证实. Mues ${ }^{[4]}$ 自己曾得到估计式

$$
\sum_{a \in \mathbf{C}} \delta\left(a, f^{(k)}\right) \leqslant \frac{k^{2}+5 k+4}{k^{2}+4 k+2} \text {. }
$$

作者 ${ }^{[6]}$ 则将上述估计改进为

$$
\begin{gathered}
\sum_{a \in \mathbf{C}} \delta\left(a, f^{(k)}\right) \leqslant \frac{2 k+2}{2 k+1} . \\
\leqslant \text { 文 部 }
\end{gathered}
$$

[1] Hayman, W. K., Meromorphic Punctions, Oxford, 1975.

[2]杨乐、值分布论及其新研究,科学出版社, 1982 .

[3] Drasin, D., An introduction to potential theory and meromorphic functions, Complex Amalysis and is Applinations, vol. 1, IAEA, Vienna, 1976, 1-93.

[ 4 ] Mues. E., Manuscripta Math, 5(1971), 275-297.

[ 5 ] Frank, G. and Weissenborn, G., Bull. London Math. Soc, 18(1986), 29-33.

[6]杨乐,科学通报,35(1990), 16: 1208-1210. 